\title{
A study on the acoustic characteristics of the sound-absorbing structure in single-column UHV Shunt reactors
}

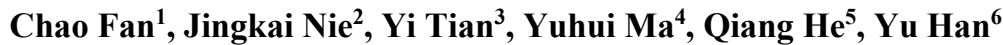 \\ $1,2,3,5,6$ Global Energy Interconnection Research Institute Co., Ltd., Beijing, China \\ ${ }^{4}$ Jinhua Power Supply Company of State Grid Zhejiang Electric Power Co., Ltd., Jinhua, China \\ ${ }^{1}$ Corresponding author \\ E-mail:117anchao006@126.com, ${ }^{2}$ niejingkai@geiri.sgcc.com.cn, ${ }^{3}$ tianyi@geiri.sgcc.com.cn, \\ 4pony_zju@126.com, ${ }^{5}$ heqiang@geiri.sgcc.com.cn, ${ }^{6}$ hanyu@geiri.sgcc.com.cn
}

Received 5 January 2022; received in revised form 15 January 2022; accepted 24 January 2022 DOI https://doi.org/10.21595/vp.2022.22388

Check for updates

Copyright $(\mathbb{C} 2022$ Chao Fan, et al. This is an open access article distributed under the Creative Commons Attribution License, which permits unrestricted use, distribution, and reproduction in any medium, provided the original work is properly cited.

\begin{abstract}
Single-column UHV shunt reactors usually generate large noises with long wavelengths. For this reason, the noises along the substation boundaries may exceed the standards. We aimed at noise control of single-column UHV shunt reactors in the present study. Based on the noise characteristics of single-column UHV shunt reactors, we employed an acoustic impedance test apparatus and studied the influences of different structural parameters on the acoustic performance of the compound impedance sound-absorbing structure. The results showed that the compound impedance sound-absorbing structure had a better sound-absorbing performance at low frequencies if a microperforated plate was introduced with basalt cotton as the filler. Besides, as the surface density, thickness, size of the dorsal cavity, and size of the intermediate cavity increased, the sound absorption coefficient at low frequencies increased. After the surface density increased to a certain level, the increase rate of the sound-absorbing performance dropped suddenly. Moreover, the intermediate cavity achieved a lesser enhancement of sound-absorbing performance at low frequencies than the dorsal cavity.
\end{abstract}

Keywords: single-column UHV shunt reactors, noises, sound-absorbing structure, compound impedance sound absorption.

\section{Introduction}

Recent years have witnessed a constant development of ultra-high-voltage (UHV) grids. However, noise pollution from UHV equipment has become increasingly aggravated [1]. The iron core of single-column UHV shunt reactors are designed as a discus, and the magnetic leakage phenomenon may be severe. In the presence of an alternating magnetic field, the attractive force between discuses and between the discus and the bracket may result in substantial vibration and noises [2]. Besides, single-column UHV shunt reactors are usually deployed along the substation boundaries. The noises generated by the reactors are predominantly in the low-frequency band and are of long wavelengths. These noises have a strong ability to penetrate the buildings. Therefore, single-column UHV shunt reactors may profoundly affect the residents in the surrounding area. Given the above, noise control of single-column UHV shunt reactors is a major concern for grid companies.

At present, glass wool and other fibers are usually used as sound-absorbing materials for single-column UHV shunt reactors. However, glass wool has a lower sound absorption coefficient at low frequencies. It is brittle, easily broken, and not environmentally friendly. Glass wool can hardly meet the noise reduction and service life requirements for single-column UHV shunt reactors [3], [4]. We performed a series of acoustic impedance tests and chose basalt cotton with smaller density, higher elastic modulus and better environmental sustainability as the filler. A compound impedance sound-absorbing structure was thus prepared. This structure could improve the sound-absorbing performance at low frequencies and widen the frequency band of sound 
absorption and therefore was applicable for noise control of single-column UHV shunt reactors.

\section{Analysis of noise characteristics}

The intensity of near-field noises generated by single-column UHV shunt reactors varies with voltage grade, rated capacity and manufacturer. Nevertheless, the intensity is generally within the range of 70 to $75 \mathrm{~dB}(\mathrm{~A})$. The frequency range of noises is mainly limited to the low frequencies, with a significant peak occurring at $100 \mathrm{~Hz}$. The sound pressure level gradually decreases after the peak. The single-column UHV shunt reactor is shown in Fig. 1, and the frequency spectrum of the generated noises is given in Fig. 2.

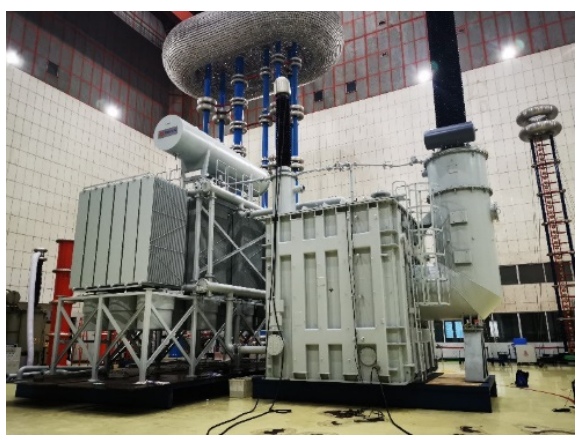

Fig. 1. Single-column UHV shunt reactor

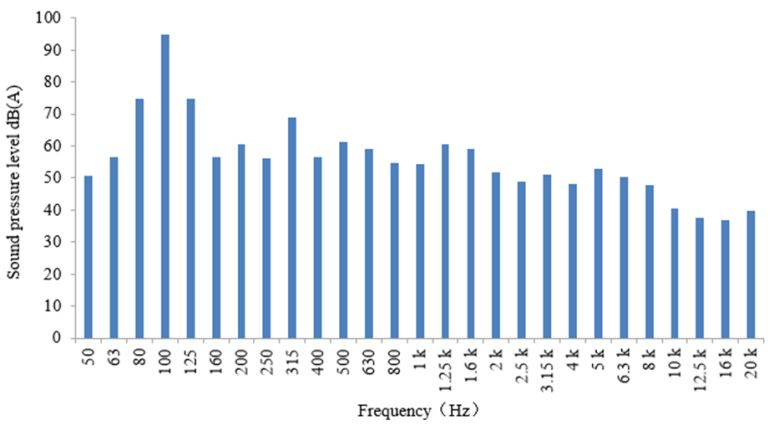

Fig. 2. Frequency spectrum of noises in a single-column UHV shunt reactor

\section{Experimental protocol}

We adopted the compound impedance sound-absorbing principle (compounding porous fibers with resonance absorber) to optimize a noise-absorbing structure suitable for single-column UHV shunt reactors [5], [6]. To achieve this goal, we first studied the influences of the type, surface density and thickness of sound-absorbing materials on the acoustic performance of the sound-absorbing structure according to GB/T 18696.2-2002 Acoustics: Determination of sound absorption coefficient and impedance in impedance tubes-Part 2: Transfer function method. We also examined the influence of the compounding parameters (namely, compounding method, size of the dorsal cavity, and size of the intermediate cavity) on the acoustic performance of the sound-absorbing structure [7], [8]. The acoustic impedance test apparatus was used for the analysis, as shown in Fig. 3. The test samples included glass wool, basalt cotton, aluminum silicate fiber cotton, and microperforated plate, as shown in Fig. 4.

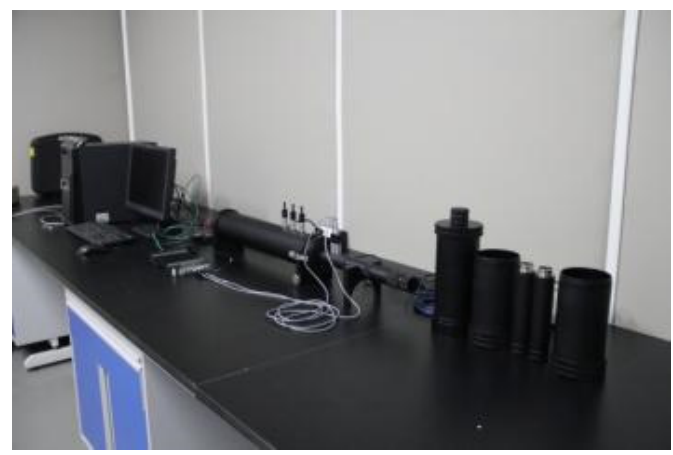

Fig. 3. Acoustic impedance test apparatus 


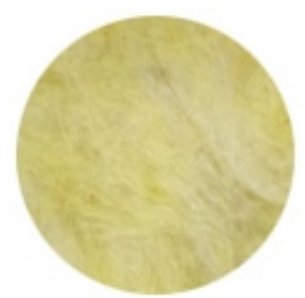

a) Cotton wool

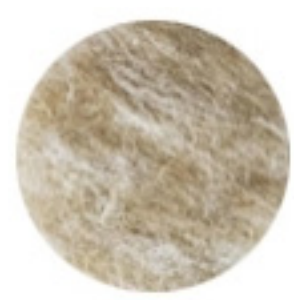

b) Basalt cotton

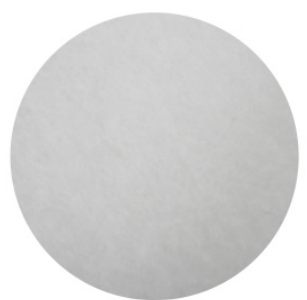

c) Aluminum silicate

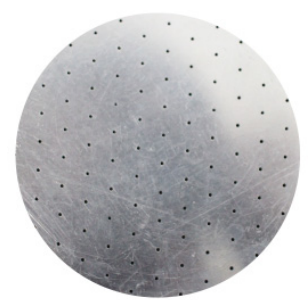

d) Microperforated plate fiber cotton

Fig. 4. Test samples

\section{Influence of material and structural parameters}

\subsection{Influence of material type on acoustic performance}

Different types of sound-absorbing materials were experimented, namely, glass wool, basalt cotton and aluminum silicate fiber cotton. Sound absorption performance was analyzed and compared among different types of sound-absorbing materials. The results are shown in Fig. 5.

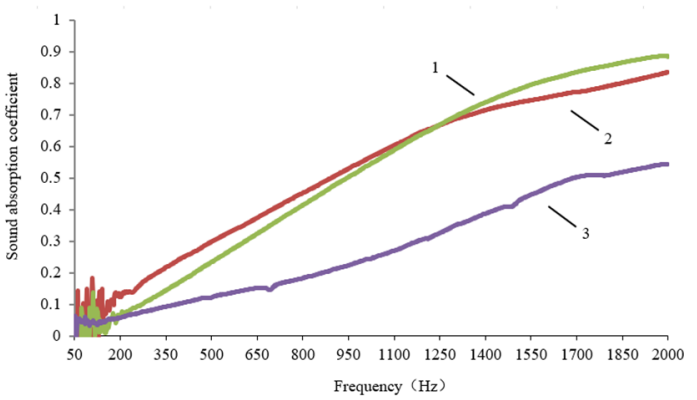

Fig. 5. Sound absorption coefficient curves of different types of sound-absorbing materials: 1 - glass wool; 2 - basalt cotton; 3 - aluminum silicate fiber cotton

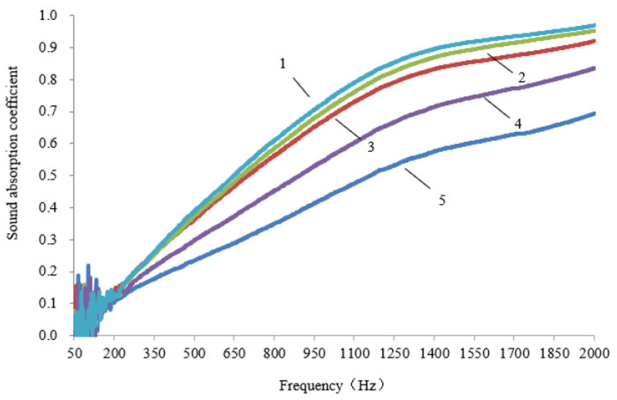

Fig. 6. Sound absorption coefficient curves of basalt cotton with different surface densities: $1-900 \mathrm{~g} / \mathrm{m}^{2} ; 2-700 \mathrm{~g} / \mathrm{m}^{2} ; 3-500 \mathrm{~g} / \mathrm{m}^{2}$; $4-300 \mathrm{~g} / \mathrm{m}^{2} ; 5-100 \mathrm{~g} / \mathrm{m}^{2}$

It can be seen from the figure that the sound absorption coefficient was the highest with basalt cotton at frequencies below $1200 \mathrm{~Hz}$. This result could be explained by the differences in density, which is $2.62 \mathrm{~g} / \mathrm{cm}^{3}$ for glass wool, $2.56 \mathrm{~g} / \mathrm{cm}^{3}$ for basalt cotton and $2.92 \mathrm{~g} / \mathrm{cm}^{3}$ for aluminum silicate fiber cotton. With all other size parameters being the same, the basalt cotton had smaller pores inside, resulting in higher low-frequency noise retention capability.

\subsection{Influence of surface density of materials on acoustic performance}

Next, we tested the sound absorption performance of basalt cotton with different surface densities: $100 \mathrm{~g} / \mathrm{m}^{2}, 300 \mathrm{~g} / \mathrm{m}^{2}, 500 \mathrm{~g} / \mathrm{m}^{2}, 700 \mathrm{~g} / \mathrm{m}^{2}$, and $900 \mathrm{~g} / \mathrm{m}^{2}$. The test results are shown in Fig. 6.

It can be seen from the figure that as the surface density increased, the sound absorption coefficient of the basalt cotton increased. When the surface density increased to $500 \mathrm{~g} / \mathrm{m}^{2}$, the sound absorption coefficient increased at a much lower rate as the surface density further increased. This was because as the surface density increased, the size of the pores within the basalt cotton reduced. As a result, the resistance encountered by the sound waves passing through the fiber cotton increased gradually. Once the surface density increased to a certain level, it was not conducive to sound absorption. This directly resulted in a sudden reduction in the increase rate of the sound absorption coefficient curve. 


\subsection{Influence of material thickness on acoustic performance}

The sound absorption performance of basalt cotton with different surface densities was analyzed, namely, $2.5 \mathrm{~cm}, 5 \mathrm{~cm}$ and $10 \mathrm{~cm}$. The test results are shown in Fig. 7 .

It can be seen from the figure that as the material thickness increased, the peak of sound absorption shifted toward the left, which was accompanied by a dramatic improvement in the acoustic performance. This was because as the material thickness increased, the length of pores inside the basalt cotton also increased to match with the length of low-frequency sound waves. Therefore, the basalt cotton became more suitable for absorbing low-frequency noises.

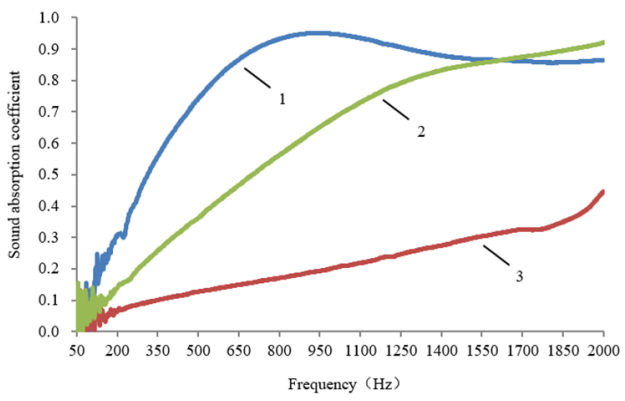

Fig. 7. Sound absorption coefficient curves of basalt cotton with different thicknesses:

$1-10 \mathrm{~cm} ; 2-5 \mathrm{~cm} ; 3-2.5 \mathrm{~cm}$

\section{Influence of compound structure parameters}

\subsection{Influence of the compounding method on acoustic performance}

A microperforated plate is a resonance absorber featured by low sound quality and high acoustic impedance, with a pore size below $1 \mathrm{~mm}$ and a perforation rate of $1-5 \%$. When the frequency of the incident sound waves is close to the resonance frequency of the microperforated plate, the air in the microperforated plate will undergo vibration and friction, causing a significant attenuation of the sound energy [9], [10].

Based on the above considerations, we used a microperforated plate as the resonance absorber in the sound-absorbing structure. The microperforated plate was placed in front of or behind the basalt cotton. The sound absorption performance was compared between the sound-absorbing structures made by different compounding methods, with the test results shown in Fig. 8.

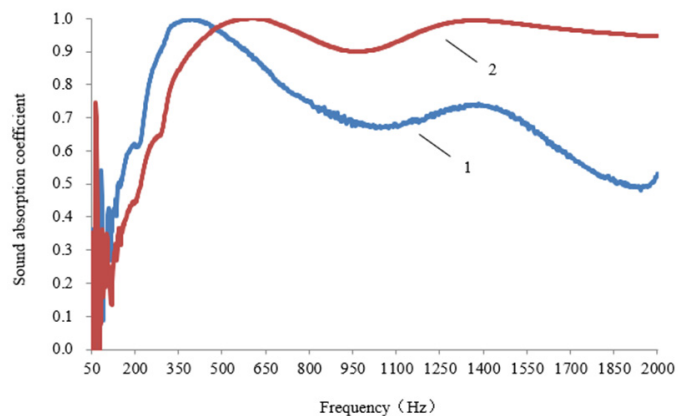

Fig. 8. Sound absorption coefficient curves of the sound-absorbing structure with different compounding methods: 1 - Microperforated plate + Basalt cotton of thickness $10 \mathrm{~cm}+$ Dorsal cavity of thickness $2 \mathrm{~cm}$;

2 -Basalt cotton of thickness $10 \mathrm{~cm}+$ Microperforated plate + Dorsal cavity of thickness $2 \mathrm{~cm}$

As shown in the figure, after adding the microperforated plates, the sound absorption coefficients of the sound-absorbing structure were significantly increased. Besides, when the 
microperforated plate was placed in front of the basalt cotton, the sound absorption coefficient at frequencies below $500 \mathrm{~Hz}$ was increased more significantly.

\subsection{Influence of the size of the dorsal cavity on acoustic performance}

With the microperforated panel as the surface layer, and the basalt cotton of thickness $5 \mathrm{~cm}$ as the filling material, different sizes of the dorsal cavity were chosen, namely, $1 \mathrm{~cm}, 3 \mathrm{~cm}, 5 \mathrm{~cm}$, and $7 \mathrm{~cm}$. The sound absorption performance of the sound-absorbing structures with the dorsal cavity in different sizes was analyzed. The test results are shown in Fig. 9.

It can be seen from the figure that as the size of the dorsal cavity increased, the sound absorption coefficient curve shifted towards lower frequencies on the whole. Therefore, the low-frequency sound absorption performance of the sound-absorbing structure was enhanced. This result could be attributed to the fact that the compounded sound-absorbing structure resembled an RLC resonant circuit, where the fiber cotton achieved a cascade connection of acoustic impedance and sound quality. The microperforated plate acted as an acoustic capacitance. When the size of the dorsal cavity matched with the wavelength of the sound waves, the sound energy could be more effectively dissipated by the dorsal cavity.

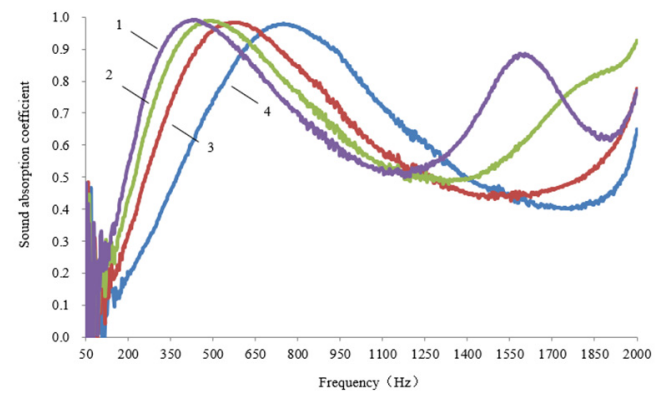

Fig. 9. Sound absorption coefficient curves of the sound-absorbing structure with the dorsal cavity in different sizes: $1-7 \mathrm{~cm} ; 2-5 \mathrm{~cm} ; 3-3 \mathrm{~cm} ; 4-1 \mathrm{~cm}$ cavity in different sizes: $1-7 \mathrm{~cm} ; 2-5 \mathrm{~cm} ; 3-3 \mathrm{~cm}$

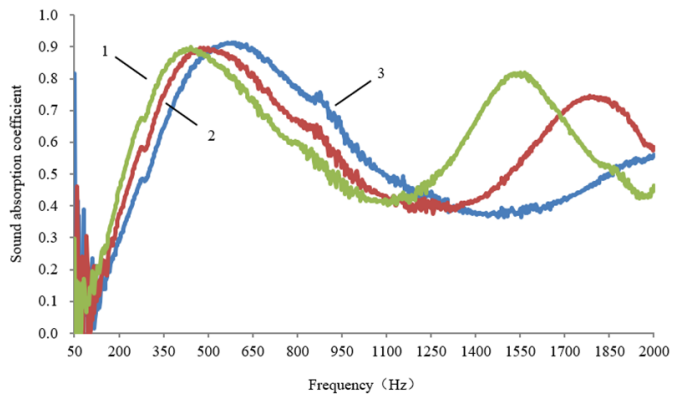

Fig. 10. Sound absorption coefficient curves of the sound-absorbing structure with the intermediate

\subsection{Influence of the size of the intermediate cavity on acoustic performance}

With the microperforated panel as the surface layer, and the basalt cotton of thickness $5 \mathrm{~cm}$ as the filling material, different sizes of the intermediate cavity were chosen, namely, $3 \mathrm{~cm}, 5 \mathrm{~cm}$, and $7 \mathrm{~cm}$. The sound absorption performance of the sound-absorbing structures with the intermediate cavity in different sizes was analyzed. The test results are shown in Fig. 10.

It can be seen from the figure that as the intermediate cavity increased in size, the sound absorption coefficient curve shifted towards lower frequencies. In other words, the increase in the intermediate cavity in size had a similar effect as that in the dorsal cavity: both were conducive to low-frequency sound absorption. But compared with the dorsal cavity, the intermediate cavity achieved a lesser enhancement of low-frequency sound absorption. This was because the basalt cotton was not backed by a cavity.

\section{Conclusions}

Based on the noise characteristics of the single-column UHV shunt reactors, we analyzed the influence of different structural parameters on the acoustic performance of the compound impedance sound-absorbing structure. We arrived at the following conclusions:

1) The noise frequency range of single-column UHV shunt reactors is mainly limited to low frequencies, with a significant peak occurring at $100 \mathrm{~Hz}$.

2) Basalt cotton had the better low-frequency sound absorption performance among the three 
materials tested. Besides, as the surface density and the thickness increased, the low-frequency sound absorption coefficient increased correspondingly. However, the increase rate dropped abruptly after the surface density increased to a certain level.

3) The addition of a microperforated plate could dramatically increase the sound absorption coefficient of the sound-absorbing structure where basalt cotton was used as fillers. Moreover, as the size of the dorsal cavity and the intermediate cavity increased, the low-frequency sound absorption coefficient of the sound-absorbing structure also increased. However, the intermediate cavity achieved a lesser enhancement of low-frequency sound absorption performance than the dorsal cavity.

\section{Acknowledgements}

Science and Technology Project of the State Grid Corporation of China Headquarters: In-Depth Research and Application of the Key Technology of Noise Reduction for Single-Column UHV Shunt Reactors (5200-201919104A-0-0-00).

\section{References}

[1] J. W. Du et al., "Measurement and evaluation ambient noise in UHV substations," (in Chinese), Noise and Vibration Control, Vol. 38, No. 6, pp. 118-123, 2018, https://doi.org/10.3969/j.issn

[2] X. Cai, Y. Sh. Ma, and Ch. Xu., "Research and control of complex sound field of high voltage reactor in UHA substations," (in Chinese), Noise and Vibration Control, Vol. 40, No. 5, pp. 187-191, 2020, https://doi.org/10.3969/j.issn.1006-1355.2020.05.032

[3] Y. Ni et al., "Test and analysis of sound insulation performance of sound insulation cover for UHV shunt reactor," (in Chinese), High Voltage Apparatus, Vol. 57, No. 2, pp. 77-83, 2021, https://doi.org/10.13296/j.1001-1609.hva.2021.02.012

[4] D. Liang et al., "Research on sound-absorption materials and structure for UHV substation noise reduction," (in Chinese), Hebei Electric Power, Vol. 37, No. 5, pp. 1-4, 2018.

[5] P. Liu et al., "A study of the new compound impedance sound-absorbing material design," (in Chinese), Environmental Science and Technology, Vol. 40, pp. 273-279, 2017, https://doi.org/10.3969/j.issn.1003-6504.2017.s2.052

[6] W. Li., "Research on sound and vibration characteristics of multi-layer acoustic structures," (in Chinese), Chongqing University, 2017.

[7] W. X. Li et al., "Measurement of inherent parameters of materials by impedance method and study on sound absorption properties of multilayer materials," (in Chinese), Noise and Vibration Control, Vol. 40, No. 3, pp. 265-269, 2020, https://doi.org/10.3969/j.issn.1006-1355.2020.03.047

[8] G. J. Ni, L. H. Lv, and X. Y. Zhao., "Preparation of sound absorption basalt fiber composite material and its properties," (in Chinese), Shanghai Textile Science and Technology, Vol. 44, No. 6, pp. 34-36, 2016, https://doi.org/10.16549/j.cnki.issn.1001-2044.2016.06.013

[9] J. X. Hou, H. Ch. Zhu, and S. W. Yuan., "Acoustic absorption characteristics of a flexible microperforated panel cavity with a flexible back one," (in Chinese), Journal of Vibration and Shock, Vol. 39, No. 15, pp. 156-162, 2020, https://doi.org/10.13465/j.cnki.jvs.2020.15.021

[10] Y. Liu, "Study on low frequency sound absorption characteristics of micro-perforated panel with multireaonance," (in Chinese), Harbin Institute of Technology, 2019. 